\title{
Clearance of materials from accelerator facilities
}

\author{
Sayed H. Rokni ${ }^{1}$, Scott L. Davis ${ }^{2}$, Ryan Ford ${ }^{1}$, James C. Liu ${ }^{1}$, Elaine Marshall ${ }^{3}$, Scott O Schwahn ${ }^{4}$ and Keith Welch ${ }^{5}$ \\ ${ }^{1}$ SLAC National Accelerator Laboratory, Menlo Park, CA 94025, U.S.A. \\ ${ }^{2}$ US Department of Energy, Germantown, MD 20874, U.S.A. \\ ${ }^{3}$ Sandia National Laboratories, Albuquerque, NM 87185, U.S.A. \\ ${ }^{4}$ Oak Ridge National Laboratory, Oak Ridge, TN 37831, U.S.A. \\ ${ }^{5}$ Thomas Jefferson National Accelerator Facility, Newport News, VA 23606, U.S.A.
}

\begin{abstract}
A new Technical Standard that supports the clearance of materials and equipment (personal property) from U.S. Department of Energy (DOE) accelerator facilities has been developed. The Standard focuses on personal property that has the potential to be radiologically impacted by accelerator operations. It addresses material clearance programs and protocols for off-site releases without restriction on use. Common metals with potential volumetric activation are of main interest with technical bases provided in Appendices of the Standard. The clearance protocols in the Standard include three elements: 1) clearance criteria, 2) process knowledge, and 3) measurement methods. This paper presents the technical aspects of the new Standard, discusses operational experience gained in clearance of materials and equipment from several accelerator facilities at SLAC and examples as to how this Standard can be applied to benefit the entirety of the DOE Accelerator Complex.
\end{abstract}

\section{Introduction}

Clearance of materials from accelerators is subject to many regulatory requirements demonstrated by the existence of international, national and regional clearance regulations or standards [1-3]. Thus, unrestricted release of materials from radiological controls can be a prohibitively restrictive process. Restrictions are generally due to: lack of accepted clearance criteria, lack of established processes to demonstrate compliance with the clearance criteria, and policies that further restrict release of materials due to public concerns, or inadequate practices. Accelerator materials and components are often subject to these restrictions despite the fact that the majority of components in accelerator facilities are not impacted by beam operations.

A new U.S. DOE Technical Standard [4] has been developed for use in DOE accelerator facilities that address these issues. The purpose of the Standard is to provide an acceptable approach for managing and dispositioning personal property that may be radiologically impacted by accelerator operations. The Standard provides criteria and guidance for accelerator facilities to develop site-specific material clearance programs (including management, technical, and operational aspects) that are in compliance with the myriade of regulatory requirements for protection of public and environment [5]. Both surface and volumetric radioactivity issues are addressed with focus on the volumetric radioactivity issues by establishing three-tier clearance criteria related to ANSI N13.12-2013 volume screening levels [3]. The appendices of the Standard provide general process knowledge, technical bases, and measurement methods for clearance of common metals with potential volumetric activation in electron and proton accelerators. The Standard leverages the large body of technical expertise and experience that exist in design and operation of accelerators [6-9], which include results of simulations by Monte Carlo radiation generation and transport codes and dedicated benchmark experiments. The technical bases and approaches used in this Standard may be applied to other types of accelerator, or even non-accelerator facilities.

\section{Activation of materials in accelerators}

Beam operation has the potential to generate radioactivity in various components in accelerators. However, in most accelerators, normal beam losses which cause material activation occur only on a limited number of components designed to intercept the full beam power (beam dumps or targets) or a fraction of the beam (e.g., collimators and septa). Other abnormal beam losses may occur at a few locations infrequently, due to mis-steering. Therefore, a large majority of materials and components in accelerator (magnets, support structures, sections of vacuum chamber) are not impacted by direct or stray radiation fields in accelerators and are good candidates for clearance

Processes leading to induced radioactivity in accelerators are well established and have been studied in detail with the use of well-benchmarked Monte Carlo codes such as FLUKA [10-11]. General process knowledge that is applicable to many accelerators can be derived from these studies. For example, the main characteristics of activation at high-energy $(\mathrm{GeV})$ electron accelerators are:

- The most abundant radionuclides are those with a half-life that is on the order of the irradiation time,

- No activation can be produced by photons with energies $<1 \mathrm{MeV}$, such as those present around klystrons and synchrotron radiation beam lines,

- No alpha emitters are produced, 
- No surface contamination is produced in metals and other solid materials, unless they are submitted to machining, drilling or grinding,

- Induced activity in any object from activation is volumetric and presents its maximum value at the surface that faces the beam loss point (surface maximum concept)

- Radioisotopes that are difficult to detect are always accompanied by "proxy" radioisotopes that can be clearly measured (proxy radionuclides concept).

The concepts of "proxy radionuclides" and "maximum surface activity" are essential for the development of measurement methods for clearance of materials with potential volumetric radioactivity (as explained in section 3.3).

The Standard provides the types of process knowledge that may allow for evaluation of volumetric induced radioactivity and its characteristics in accelerator facilities. Appendices C and D of the Standard provide the technical basis for the material clearance at high energy electron and proton accelerator facilities, respectively.

Clearance criteria and measurement methods for materials with surface radioactivity are available and standardized. Therefore, clearance of activated materials that may contain residual volumetric radioactivity in the main scope of the Standard and the main subject discussed in the following sections.

\section{Clearance Protocols}

The clearance protocols in the Standard include three elements: 1) a 3-tiered clearance criteria, 2) process knowledge, and 3) measurement methods. Process knowledge and/or measurements are used to demonstrate that the materials released meet the selected clearance criteria. Materials that have been characterized based on process knowledge evaluation and/or measurements to meet one of the clearance criteria may be released.

\subsection{Clearance criteria}

DOE Order 458.1 [5] specifies a Total Effective Dose constraint of $10 \mu \mathrm{Sv}$ above background in any calendar year for each specific clearance of personal property with potential residual radioactivity. Based on a dose constraint of $10 \mu \mathrm{Sv} / \mathrm{y}$, the ANSI N13.12 Standard [3] derives and establishes the screening levels (SLs) for various radionuclides (divided into 5 groups) for the clearance of materials that contain or may contain residual levels of surface or volume radioactivity of radioactive materials from radiological control.

The ANSI N13.12 volume SL for proxy radionuclides represents an activity level that is detectable with common, sensitive survey meters. Therefore, for clearance of property with potential volumetric radioactivity, this Standard adopts a 3-tiered clearance criteria that can be related to ANSI N13.12 volume SLs:

1) Indistinguishable from Background (IFB) at a level lower than the ANSI N13.12 volume SLs,

2) Non-IFB, but no more than the ANSI N13.12 volume SLs,

3) Higher than the ANSI N13.12 volume SLs. Release based on this clearance criterion would follow the site or operation specific standards provided for in existing DOE regulations. It may include use restrictions or designated end use for the property.

\subsubsection{Indistinguishable from Background Clearance Criterion}

The IFB clearance criterion (i.e., material has no detectable radioactivity other than background) is the lowest and most restrictive criterion. Materials meeting the IFB clearance criterion need not be subject to radiological control and may have unrestricted release. Release based on IFB criterion requires the least justification and regulatory review and should be the preferred clearance criterion. Measurements and clearance based on IFB criterion are more straightforward to be conducted than those with non-IFB clearance criteria.

The IFB level of a measurement method depends on the detection capability of the chosen method. When IFB is used as the clearance criterion, the measurement method needs to have sufficient detection capability (which is less than the ANSI N13.12 volume SLs) to provide reasonable assurance that materials and equipment released on the basis of this criterion satisfy the $10 \mu \mathrm{Sv} / \mathrm{y}$ dose constraint. The measurement methods described in the Standard are designed to meet these objectives.

\subsection{Process knowledge and its application}

General process knowledge such as beam particle type, beam energy, average power, etc., is applicable to accelerator facilities with similar characteristics and operational modes (in terms of producing induced radioactivity). Specific process knowledge includes operational information such as beam energies and beam losses that are relevant to the specific facility, location and use of equipment within the facility as well as any temporary or long-term storage decisions. Specific process knowledge should be used to support the clearance measurements and decisions.

\subsection{Measurement methods}

Radionuclides that can be produced in common metals or concrete from activation such as ${ }^{22} \mathrm{Na},{ }^{54} \mathrm{Mn},{ }^{60} \mathrm{Co},{ }^{65} \mathrm{Zn}$ and ${ }^{152} \mathrm{Eu}$ are in Group 1 radionuclides in ANSI N13.12- 
2013 Standard, as they emit high-energy and high-yield gamma rays. These proxy radionuclides have a volume $\mathrm{SL}$ of $0.1 \mathrm{~Bq} / \mathrm{g}$, that is $1 / 10$ of the previous ANSI N13.12-1999 value. For the measurements of potential volumetric radioactivity in this Standard, many of these radionuclides may serve as "proxy" radionuclides for hard to detect radionuclides, because they are easy to be measured with common survey instruments.

The concepts of "measurements of proxy radionuclides" and "maximum surface activity" serve as the technical bases for the surface survey method for volumetric radioactivity recommended by this Standard. These concepts allow for efficient material release surveys in the fields via the following measurement protocols:

- Measurements for proxy radionuclides are necessary and sufficient.

- Surface surveys for proxy radionuclides using portable instruments and techniques with high sensitivity in an environment with low background are adequate and sufficient.

- Surface surveys of all surfaces of an object, regardless of its shape and size, are sufficient. Measurement of only the surface of an object that faces the beam loss point is also acceptable, if the beam loss geometry is known.

The detection capability needs to be evaluated and documented based on the instrument's sensitivity to proxy radionuclides in the materials and the acceptable background in the measurement environment. The sensitivity depends on factors such as the material type, types of radionuclides, volumetric radioactivity distribution, the detector-sample geometry, and the measurement mode such as scanning or fixed-position, etc. The minimum sensitivity and appropriate background level should be used such that the detection capability can be estimated conservatively. The Standard provides examples of appropriate instruments and techniques and estimations of associated detection capabilities. Many sensitive, portable instruments that are commercially available (e.g., scintillator-based survey meters) have capability to detect the proxy radionuclides at levels that are less than the corresponding ANSI N13.12 SL values.

\section{Operational experience}

The clearance program requirements and protocols prescribed by the DOE Technical Standard have been used for recycling and reuse of large amounts of metals at several DOE accelerator facilities. At SLAC, approximately 2,000 tons of metals have been recycled from Positron Electron Project accelerators and the BaBar detector, SPEAR-II and FFTB since 2011. The types of materials released include cables, pipes, magnets, vacuum chambers and support structures for the accelerators and detectors. These recycling projects create values to DOE and SLAC and avoid costs of disposing the metals as radioactive waste.
Only materials that meet the IFB clearance criterion have been released for recycling at SLAC. Materials from accelerator housings are surveyed for both surface contamination and volumetric activation using practical and sensitive field survey instruments with detection capabilities that are a few times lower than the ANSI N13.12 volume SLs. The measurements using sensitive field survey instruments are supplemented by more sensitive confirmatory measurements such as high resolution field gamma-ray spectrometry. SLAC measurement instruments and methods have sufficiently low detection capabilities that satisfy the requirements in this Standard and are lower than the ANSI N13.12 Screening Levels which correspond to a dose based limit of less than $10 \mu \mathrm{Sv} / \mathrm{y}$.

\section{References}

1. IAEA, "Clearance Levels for Radionuclides in Solid Materials", International Atomic Energy Agency (IAEA), Vienna, Austria, IAEA-TECDOC-855, (1996)

2. NCRP, "Managing Potentially Radioactive Scrap Metal," National Council on Radiation Protection and Measurements (NCRP), Bethesda MD, NCRP Report No. 144, (2002)

3. ANSI, "Surface and Volume Radioactivity Standards for Clearance", American National Standard Institute (ANSI), ANSI N13.12, (2013)

4. U.S. DOE, "Clearance and Release of Personal Property from Accelerator Facilities", U.S. Department of Energy Standard, in review and approval process (2016)

5. U.S. DOE, "Radiation Protection of the Public and the Environment", Department of Energy Order 458.1, Chg. 3, (2013)

6. SLAC, "SLAC Material Release Program Manual", SLAC National Accelerator Laboratory (SLAC), Radiation Protection Department procedure RPD010, (2011)

7. JLAB, "Technical Basis for Release of Solid Materials from Radiological Control when Residual Radioactivity Levels Are Indistinguishable from Background," Thomas Jefferson National Accelerator Facility (JLAB), Radiation Control Department, (2009)

8. A. H. Sullivan, "A Guide to Radiation and Radioactivity Levels near High Energy Particle Accelerators," Nuclear Technology Publishing, ISBN 1-870965-18-3, Ashford, Kent, England, (1992)

9. K. Masumoto, H. Matsumura, K. Bessho, and A. Toyoda, "Role of Activation Analysis for Radiation Control in Accelerator Facilities." Journal of Radioanalytical and Nuclear Chemistry, 278, No.2, 449-453, (2008) 
10. T. Böhlen, F. Cerutti, M.P.W. Chin, A. Fassò, A. Ferrari, P.G. Ortega, A. Mairani, P.R. Sala, G. Smirnov and V. Vlachoudis, The FLUKA Code: Developments and Challenges for High Energy and Medical Applications", Nuclear Data Sheets 120, 211-214 (2014)

11. A. Ferrari, P.R. Sala, A. Fasso', and J. Ranft, "FLUKA: a multi-particle transport code", CERN2005-10 (2005), INFN/TC_05/11, SLAC-R-773

*This work is supported by the U.S. DOE, Office of Science, Office of Basic Energy Sciences, under Contract No. DE-AC02-76SF00515. 